Article

\title{
Flooding Message Mitigation of Wireless Content Centric Networking for Last-Mile Smart-Grid ${ }^{\dagger}$
}

\author{
Jaebeom Kim *, Byung-Seok Park and Yong-up Park \\ KEPCO Research Institute, Smart Distribution Laboratory, Munji-ro 105, Yu-seong gu, Daejeon 34056, Korea; \\ blud-grid@kepco.co.kr (B.-S.P.); yu.park@kepco.co.kr (Y.-u.P.) \\ * Correspondence: jaebeom@kepco.co.kr \\ + This paper is an extended version of paper published in the 19th IEEE International Conference on Networks \\ (ICON), Singapore, 11-13 December 2013.
}

Received: 16 July 2019; Accepted: 17 September 2019; Published: 23 September 2019

Featured Application: Smart Grid network; Industrial 4.0 network.

\begin{abstract}
In view of Smart-Grid architecture, wireless Last-Mile Network (LMN) devices as smart meters and intelligent home control machines are normally installed in harsh and lossy communication environment. In order to improve communication reliability of LMN, we proposed Wireless Topology Aware Content Centric Networking (TOP-CCN) protocol. TOP-CCN reduces channel access overhead of traditional Content Centric Networking and supports efficient multicast message transmission by using Multiple Point Relay (MPR), and Publisher MPR (PMPR). In addition, TOP-CCN LMN provides simple multi-hop forwarding scheme that can reduce the traditional routing control message overhead in multi-hop wireless LMN environment. The simulation result shows TOP-CCN can improve the service provisioning time and reliability compared to traditional IP based network model in LMN.
\end{abstract}

Keywords: Advanced Metering Infrastructure (AMI)network; internet-of-things; wireless content centric networking; last-mile smart grid

\section{Introduction}

Power grid management strategy, such as Smart Grid (SG), is rapidly evolving with ICT paradigm. In the SG, reliable smart metering data transmission has to be guaranteed to calculate control power usage, grid load, price, power generation control, etc. The Last-Mile smart grid Network (LMN) is edge area system of SG. The LMN is designed to collect raw power usage data of electricity consumers and has to process control traffic between SG server and distributed energy resource (DER) devices. Therefore, it has to be robust and cost-effective. In an LMN network, each device periodically generates and transmits a metering data to server and server eventually sends a regional data requests packet. LMN application generally includes remote firmware update, power price update, time-of-use, peak-load data, etc. [1]. In LMN, Power-Line-Communication (PLC) and Wi-SUN communication modems are widely utilized to reduce the infrastructure cost. However, network capacity is strictly designed because PLC and Wi-SUN only provides several kb/s network capacities [2]. Thus, the network efficiency is most critical issues. RPL, 6LowPAN, IP multicasting can be utilized to improve network efficiency of LMN. However, network stack complexity and overhead such as IP address assignment, routing table management, header compression processing overhead, and various problems have to be optimized [3].

Content Centric Networking (CCN) is one of the solutions to improve LMN communication reliability [4]. CCN is non-IP based communication model and it has recently gained significant 
recognition for its abilities to provide efficient end-to-end data dissemination without traditional IP address management overhead. From these advantages, $\mathrm{CCN}$ is already being exploited in many kinds of wireless applications and services and bound to be utilized in future networking such as Internet-of-things (IoT) [5]. In CCN LMN, each meter device can create a unique content as metering data of each household and can be a content provider. A device who want to receive a specific data transmits an Interest packet with unique, persistent, location-independent name. This interest packet is flooded to all network to make backward path to content requester for a certain time. When a $\mathrm{CCN}$ device receives Interest packet from content requester device, it looks for a suitable content in its own cache. If the device finds a related content, then the content will be directly transmitted to content requester via backward path. Each device in a backward path can cache received content packet to send content to requester instead of content originator when a device receives same Interest packet. Especially, CCN can effectively support to broadcast and multicast data dissemination for data offloading of the server. However, the generic form of CCN may also have flaws of its own in wireless $\mathrm{LMN}$ environment due to broadcast storming problem because all types of $\mathrm{CCN}$ packets are flooded to local network.

Wired communication protocols are mandatory to enabling stable AMI system but its installation cost have to be optimized. Power-line-communication protocols are widely installed in AMI LMN environment but that frequently shows instable performance depending on background noise of the power line due to customer's power usage, and power grid network design. To improve smart metering service stability, wireless communication protocols are considered to LMN environment. LTE system is very stable that is widely applied to back-end network area between DCU and Server. However, in wireless LMN environment, there are many candidate long-range wireless communication protocols such as IEEE 802.11ah, SixFox, NBIoT, LoRa, and IEEE 802.15.4-2015 SUN (a.k.a IEEE 802.15.4g) [6]. SisFox and Lora communication range is longer than IEEE 802.15.4-2015 SUN that can communication few kilometers, but data rate shows only few bps. IEEE 802.15.4-2015 and NB-IoT shows $1 \mathrm{~km}$ communication range and up to several hundred data rates, but in high-density AMI environment, packet error rate is increased because most of smart meter devices are installed in closed steel box and plastic cages [6]. Thus, the multi-hop routing functionalities have to be considered for wireless LMN environment.

In this paper, we propose a Wireless Topology Aware Content Centric Networking (TOP-CCN) for LMN. In our previous work, we focused on the mobile ad-hoc network environments and voice and video traffic delivery. Different from our previous work, we proposed Smart Grid content naming, system model, and content delivery architecture in this paper. Also, this proposed architecture focused on network heterogeneity as IP and CCN mixed environment to guarantee interoperability between Smart Grid standard application protocols such as DLMS/COSEM, and CoAP. Flooding mitigation and reduction in layer 2 communication, TOP-CCN employs Multiple Point Relay (MPR) based packet forwarding, publisher MPR (PMPR) based proactive content discovery, and flooding range control algorithms based on 1-hop and 2-hop neighbor information. In TOP-CCN algorithm, every meter device periodically elects its MPR, and PMPR through 1-hop broadcast content announcement message. Multi-hop content announcement message from the selected PMPR is broadcasted through MPR to reduce the number of flooding messages of the LMN. Based on a simulation study using ns-3 [5], we show the improved performances of our proposal in terms of communication reliability, network overhead, content downloading time compared to related work.

\section{System Model and Related Work}

\subsection{System Model}

Content Centric Network does not require IP addressing to access data in a specific network. Instead of IP address, CCN uses Content-prefix and Face information. The content-prefix is unique content identifier that can be created by naming rules. For example, in smart grid network, it can 
be modeled by URI structures as following application parameters as DLMS data structure [3]. (e.g., 'MeterType/MeterID/peakLoadData/'). A Face is logical communication interface name of content-prefix that can be a realized as MAC address, network card number, etc. In a CCN, each device maintains three types of table as Forwarding Information Base (FIB), Pending Interest Table (PIT), and Content Store (CS). These tables are updated by exchanging Content-prefix Announcement (CA), Interest, and Content packet.

The FIB consists of content-prefix and Face(s) information. When a device receives Content or Interest packet, then content-prefix and related in/out bound Face are recorded to FIB. The PIT is updated by Interest packet. If a device generates or receives an Interest packet, then related content-prefix and in/out bound Face(s) are inserted to PIT table. The CS is a cache list table that is maintained eventually during exchanging Content packet. When a device received Interest packet, device lookups the CS to find a related content. If the device has the related Content, the Interest packet flooding shall be stopped, and Content packet is transmitted toward content requester through outbound Face. This means that content packet can be transmitted by each device instead of content originator to reduce the end-to-end transmission overhead. Thus, TOP-CCN provides in-network caching functionality to achieve efficient content delivery in LMN [1]. The CCN-based smart grid network can eliminate IP complexity problems. Thus, CCN may improve LMN communication efficiency by using simple architecture, temporal data caching and name-based discovery functions [7-11]. Also, CCN can reduce transmission overhead by the in-network caching strategy. In a $\mathrm{CCN}$ smart grid environment the content packet is stored to CS of the device to ensure content cache effect.

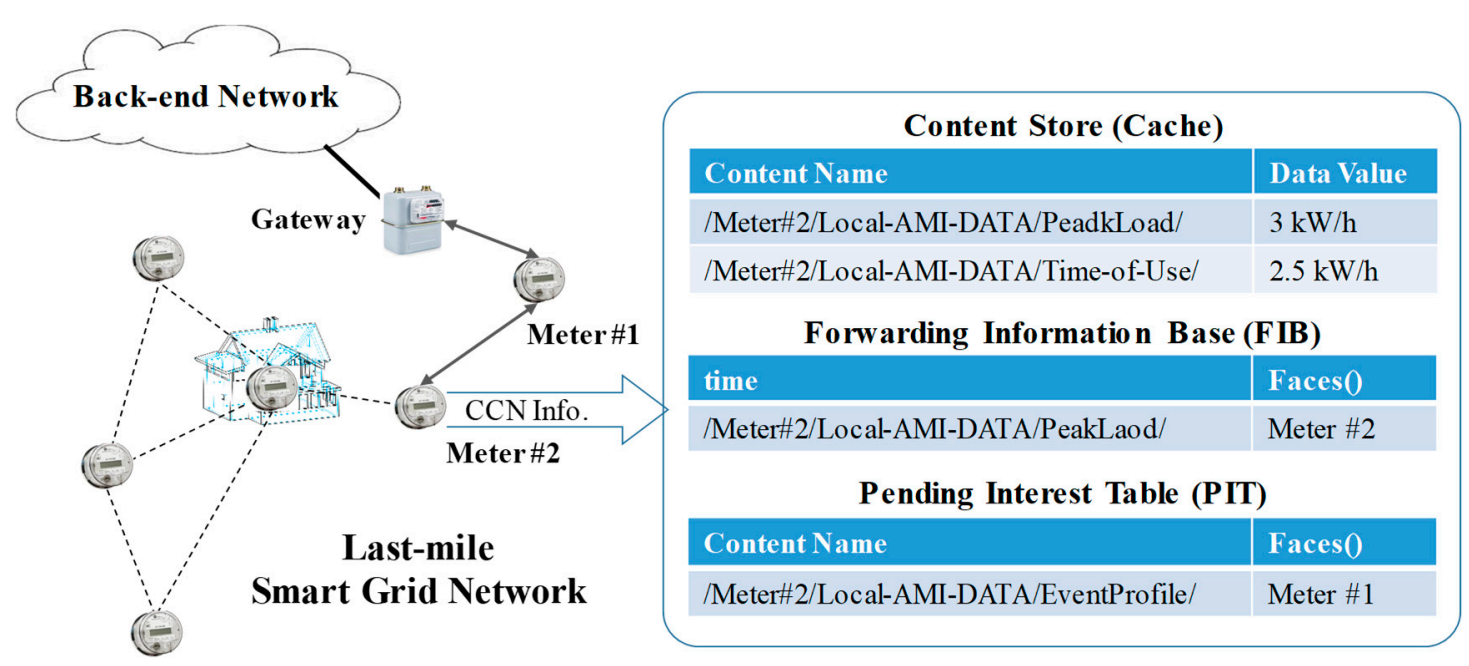

Figure 1. System model of Content Centric Networking (CCN)-based Last-mile Smart Grid Network for Advanced Metering Infrastructure.

Figure 1 shows a Wireless CCN architecture for Smart Grid LMN. In traditional AMI system, Data Concentrate Unit (DCU) is located in each LMN. In our system model, DCU or gateway uses IP stack and non-IP based CCN stack for packet routing between different network domain between back-end communication network and $\mathrm{CCN}$ based LMN. If a gateway received data sending request of Meter\#2 from IP server and has to prepare related data collection and sending. To collect requested data, gateway broadcasts an CCN Interest packet to network. Interest packet is broadcasted to network and each meter device updates PIT table using received requested content name and Face information as Interest sender's MAC address. When a device finds a requested content from its CS, then content will be transmitted to related Face(s) in a FIB. To reduce interest packet broadcast, each CCN meter device can announce its unique content list by using Content-prefix Announcement (CA) packet. If the device receives CA, CA information and outgoing Face to contents originator is recorded to FIB. If a device already knows face information of requested interest packet, then interest packet is transmitted to dedicated Face(s). The Content packet is an actual data packet related to interest packet. The FIB 
can be updated by various approaches such as proactive CA, reactive content discovery schemes, or manual setup by network managers [11]. As describe above, IP address is not utilized in a CCN because content data can be stored in various location in a local network and IP address can be replaced to specific content prefix.

\subsection{Related Work}

$\mathrm{K} \mathrm{Yu}$ et al. proposed a smart grid $\mathrm{CCN}$ architecture, and naming policy for smart metering service [3]. In the proposed architecture, authors define CCN Smart Grid attribute unit based on traditional Smart Metering data format as DLMS/COSEM and create basic URI form for Smart Grid CCN data exchange. K Kumar proposed publisher-subscriber based SG network for wide area monitoring environment [10]. In the proposed scheme, the downwards traffic of the SG system such as critical price alert, firmware, metering request contents are propagated to LMN from the control server of the Smart Grid. To reduce the content propagation delay, rendezvous device that has the highest centrality of the network store the data from the SG server. Thus, the end-to-end distance between server and AMI devices, downloading time, and data propagation time are decreased compared to Host-centric (IP based) SG network. However, these algorithms did not consider wireless LMN environment.

Wireless CCN algorithms can be adapted to LMN environment. Soon Y. Oh designed a CCN for tactical and emergency MANETs [12], called MANET CCN. To solve broadcast storming problem, MANET CCN utilizes the overhearing mechanism. When a node overhears a neighbor's interest packet, then the node aggregates the interest information. In addition, content reply mechanisms help to avoid packet collision. There are two types of messages, such as Reply and Request. A provider sends a Reply in response to the interest packet and waits for a Request from the consumer before delivering the content. In MANET CCN, content items can be classified as situation based (e.g., temporal and spatial) items and topic-based content items. Situation content items have higher priority than topic content items because they include location and time information. Thus, situation content items are quickly disseminated even though there are no request.

M. Amadeo et al. present a CCN based MANET protocol named E-CHANET that performs reactive based routing, forwarding, and reliable transport functions [13]. In contrast with above algorithms, E-CHANET tries to solve not only the broadcast storming problem but also the channel unreliability and disruption through the extended content request and delivery procedures of the pure CCN. In E-CHANET uses counter-based suppression mechanism before the packet transmission. When a node hears the same packet that has the same content prefix or content item, then packet is discarded for eliminating duplicated transmission. P. Sharma et al. [14] proposed a MANET-aware CCN algorithm on Android smartphones using Wi-Fi and Optimized Link State Routing (OLSR). This algorithm focuses on the message dissemination efficiency in highly mobile environments. To provide the higher probability of message delivery, MANET-aware CCN selects MPRs as content forwarding delegate. MPR can provide higher and optimized reachability to other nodes. MANET-aware CCN is based on CCN overlays because it brings the MANET optimizations for neighbor node reachability. Authors implemented two CCN overlays over OLSR using Haggle. For the first overlay, they kept Haggle's native algorithm for message forwarding. However, they removed link sensing and neighbor discovery function from Haggle CCN because OLSR already provides these functions. For the second overlay, the first overlay is extended by proposed MPR-aware forwarding algorithm. However, MANET-aware $\mathrm{CCN}$ uses the IP address to maintain neighbor information.

\section{Proposed Scheme}

TOP-CCN is designed to reduce the broadcast storming problem and to enhance the communication stability of CCN for LMN. Figure 2 shows the protocol stack of the TOP-CCN enabled wireless LMN. In this figure, all devices are connected with AMI gateway and gateway is connected to SG server. If the local gateway receives the metering data request traffic such as peak price alert or firmware update content from the SG server, then the gateway stores and announces the content information using 
CA packet. The content is stored by the SG gateway until a SG device requests the content through interest packet transmission, which is then disseminated to the requestors and potential devices that may cache the content for shorter-length data delivery. Each device processes the smart grid traffic using smart grid application protocol such as IEC 61805, open smart grid protocol, and ANSI C12.18 table structure [15]. In the proposed TOP-CCN architecture, each application traffic is classified by the content classifier. Basically, the network management traffic is processed by legacy IP stack. However, LMN application traffic such as DLMS can be translated to TOP-CCN.

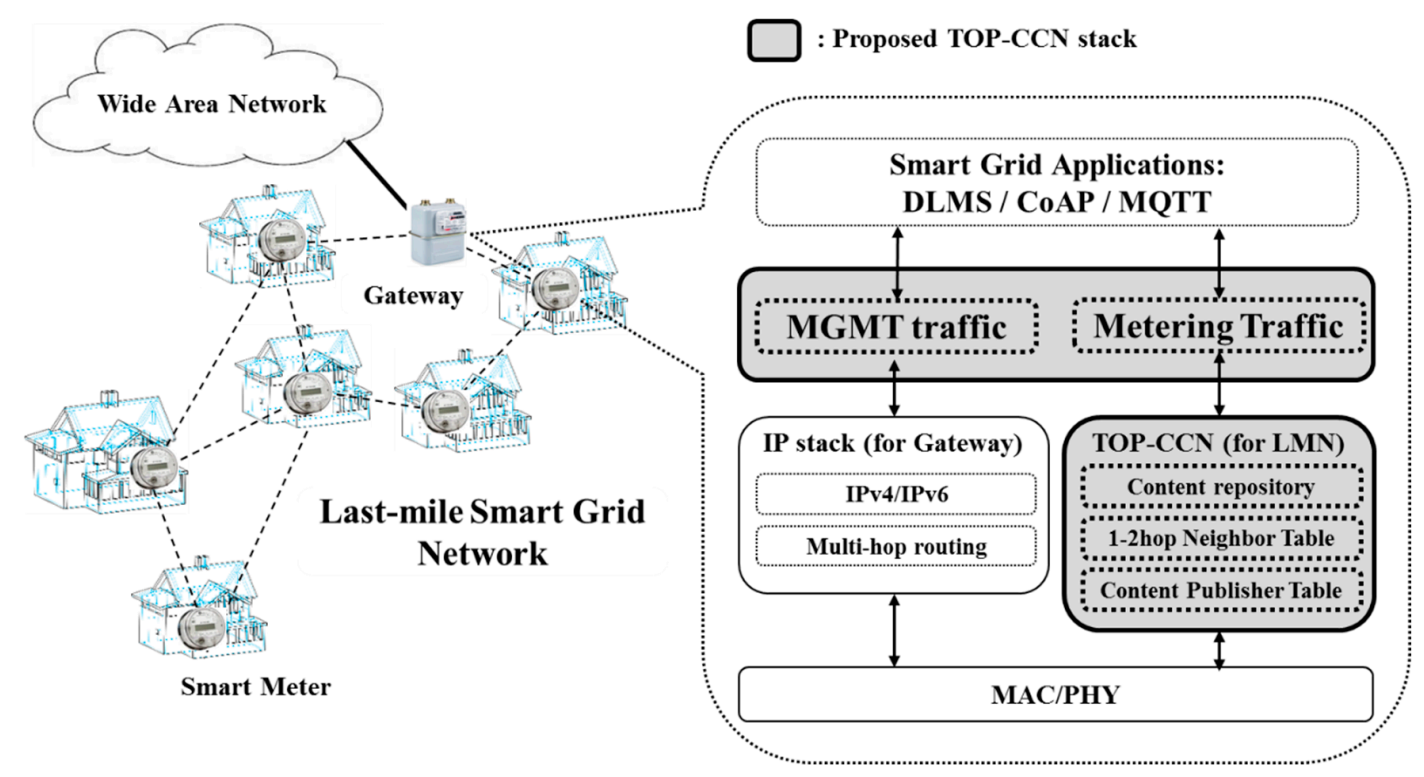

Figure 2. Network design and stack architecture of the Smart Grid Last-Mile Network (LMN).

Figure 3 is an operation example of CCN in LMN. Requested Event profile (e.g., peak power data) of all meters from server side is translated to CCN interest packet instead of IP format in a gateway. Interest packet is propagated to LMN. For example, if the server requests a regional event profile information of all meters, 'Local-AMI-DATA/EventProfile/*or All_Device/' interest packet is broadcasted to network. Interest message is propagated to network and each device update its PIT to make simple backward routing path. Thus, content packet can be transmitted to server using backward path without any additional routing algorithms.

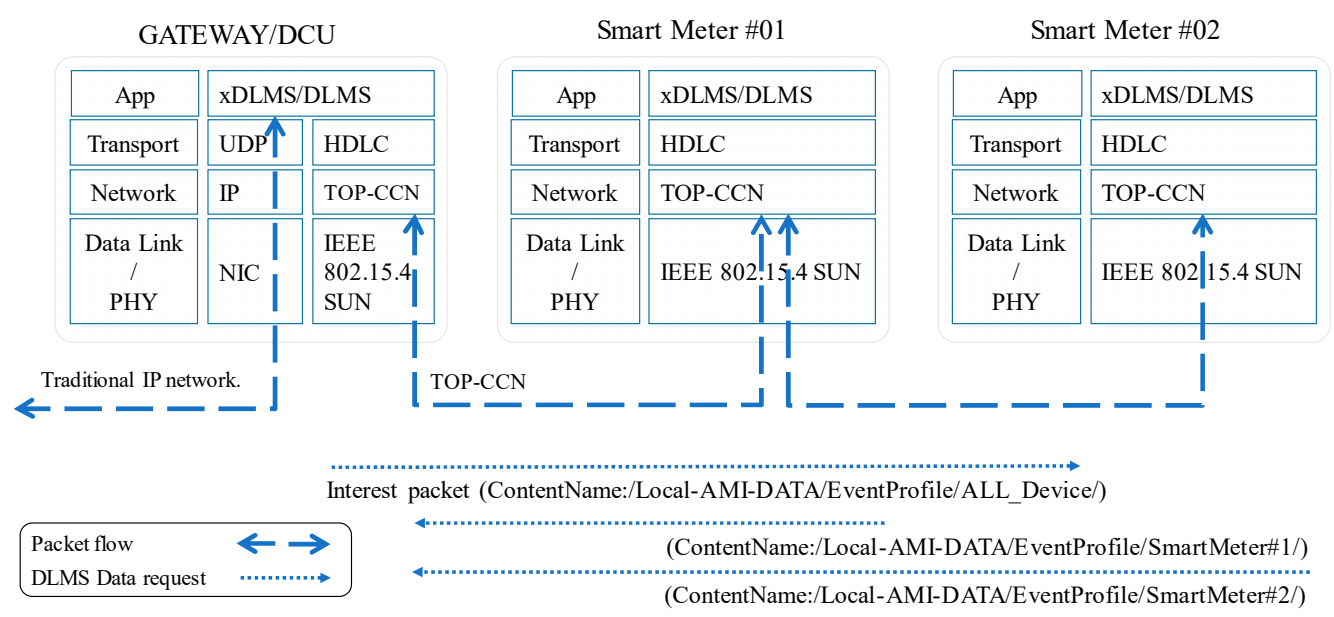

Figure 3. An operation example of Topology Aware Content Centric Networking (TOP-CCN) for LMN. 


\subsection{Extended CCN Packet Formats}

TOP-CCN extends the traditional CCN header formats to include the topology information and application characteristics of the SG application. We define three formats, CA packet, data packet, and the interest packet as shown in Figure 4. CA has ten fields; content prefix, time, ID, type, number of neighbors (\# of NBRs), neighbor prefix list (NBR-PL), neighbor list (NBR-L), neighbor type list (NBR-TL), HOP count, cache hit ratio, and CA type. Content prefix is the name of the content provider that is assigned by the network manager or the prefix assignment algorithm [3] as '/region/MeterID/eventType/Information'. Time field represents the available time duration of the stored content that can be assigned by application layer protocol. The ID is a unique identifier of the device such as MAC address or assigned meter device ID. Type attribute represents the type of the relay policy of the device. Its default value is non-MPR, which means that the content and control packets cannot be rebroadcasted. If some device is elected as a MPR by neighbor(s), then the device can rebroadcast the received TOP-CCN packet, and its type attribute is set to MPR notifying the flooding policy of the device. NBR-L is a ID list of the neighbor(s) gathered by the received CA message from neighbor(s). NBR-TL represents the type list of neighbor(s) such as MPR or not. The number of NBR fields represent to included number of neighbor list in the packet to decide the neighbor related field size in the packet. NBR-PL is a list of content prefixes of the entire neighbors. HOP count is the forwarded count of the packet that is increased by each forwarding device. Every device periodically measures a cache hit ratio during a period of time $\Delta \mathrm{t}$. Cache-hit ratio is shown as the content reply ratio among the entire requested content items. When a device has fresh or popular content items in its CS, then the cache-hit ratio will be measured as a high value. When a device receives a CA packet from one of its neighbors, then the 1-hop neighbor table, the 2-hop neighbor table, and the FIB are sequentially updated. CA type attribute represents the type of the flooding policy of the CA message such as 1-hop limit and multi-hop flooding. Both interest and content packets of the TOP-CCN have three extended fields such as the ID, expected hop, and the hop count. ID is the unique identifier of the sender such as consumer or provider of the packet. The expected hop represents the distance between the consumer and the content provider. The expected hop in the interest packet and the content packet are updated by the sender and relay devices to limit the flooding range of the packet.

\begin{tabular}{|c|c|c|c|c|c|c|c|c|c|c|c|c|}
\hline variable & Bits:48 & & 1 & 1 & 6 & & variable & 16 & 1 & $\ldots$ & 8 & 8 \\
\hline $\begin{array}{c}\text { Content } \\
\text { name }\end{array}$ & ID & & $\begin{array}{l}\text { vice } \\
\text { pe }\end{array}$ & $\begin{array}{l}\text { CA } \\
\text { type }\end{array}$ & $\#$ of $\mathrm{NI}$ & & NBR-PL & NBR-L & NBR-TL & $\cdots$ & $\begin{array}{c}\text { Hop } \\
\text { Count }\end{array}$ & $\begin{array}{c}\text { Cache } \\
\text { hit Ratio }\end{array}$ \\
\hline \multicolumn{13}{|c|}{ Interest packet format: } \\
\hline \multicolumn{5}{|c|}{$\leftarrow$ size: depending on $\mathrm{CCN}$ version $\rightarrow$} & Bits:48 & & 8 & 8 & & & & \\
\hline $\begin{array}{c}\text { Content } \\
\text { name }\end{array}$ & \multicolumn{2}{|c|}{ selector } & \multicolumn{2}{|c|}{ nonce } & *ID & & $\begin{array}{l}\text { xpected } \\
\text { Hop }\end{array}$ & *Hop Count & & & & \\
\hline \multicolumn{13}{|c|}{ Content object (data) packet format: } \\
\hline \multicolumn{5}{|c|}{$\leftarrow$ size: depending on $\mathrm{CCN}$ version $\rightarrow$} & Bits:48 & & 8 & 8 & 8 & & Variable & \\
\hline $\begin{array}{c}\text { Content } \\
\text { name }\end{array}$ & \multicolumn{2}{|c|}{ Signature } & \multicolumn{2}{|c|}{ Sign info } & $*$ ID & & $\begin{array}{l}\text { xpected } \\
\text { Hop }\end{array}$ & *Hop Count & $\begin{array}{c}* \text { Payload } \\
\text { Length }\end{array}$ & & $\begin{array}{l}* \text { Content } \\
\text { payload }\end{array}$ & \\
\hline
\end{tabular}

Figure 4. Frame format of the TOP-CCN, *: extended or new elements of the TOP-CCN compared to pure CCN [2].

\subsection{Efficient CCN Packet Dissemination by MPR Devices}

TOP-CCN defines the two tables for maintaining 1-hop and 2-hop neighbor information, respectively. The 1-hop neighbor table contains ID and lifetime of the device, whereas the 2-hop neighbor table includes 2-hop neighbor ID, connected 1-hop neighbor ID, and lifetime of the 2-hop neighbor. Both tables are periodically updated from the information of the received CA packets. The lifetime of the neighbor information is periodically initialized by CA message from the neighbor. When a lifetime of neighbor is expired, then the relative 1-hop and 2-hop neighbor information 
is removed. In addition, face information in the FIB and PIT is removed to prevent any wrong content delivery.

In CSMA/CA based wireless environments, broadcast transmissions may induce potential problems such as data congestion and packet collision due to shared medium characteristics [16]. However, $\mathrm{CCN}$ only considers the broadcasting approach to transmit packets, which is regarded to be especially error-prone to these environments. To mitigate the collision and congestion problem in CCN over wireless environment, TOP-CCN selects a MPR set that can disseminate its received packets such as interest, content, and multi-hop CA packets. If a device that is not elected to MPR or PMPR then cannot rebroadcast of dissemination packets. The MPR is elected by neighbor devices. To select a MPR, every device periodically calculates MPRcost using Equation (1),

$$
\text { MPRcost }_{i}=(1-a) 2 \text { hRatio }_{i}+\text { CacheHitRatio }_{i}(0 \leq \mathrm{a} \leq 1)
$$

where $\mathrm{i}$ is the neighbor number index, $2 \mathrm{hRatio}_{\mathrm{i}}$ represents the ratio of the 2-hop neighbor connectivity ratio of the neighbor ${ }_{i}$. For example, a device has six 2-hop neighbors and its neighbor ${ }_{i}$ has three neighbors, then $2 \mathrm{hRatio}$ of interface $\mathrm{i}_{\mathrm{i}}$ is 0.5 . If a neighbor ${ }_{i}$ has the same number of neighbors as the number of 2-hop neighbors of the device, $2 \mathrm{hRatio} i \mathrm{is}{ }_{1}$. CachehitRatio is given by CA packet from

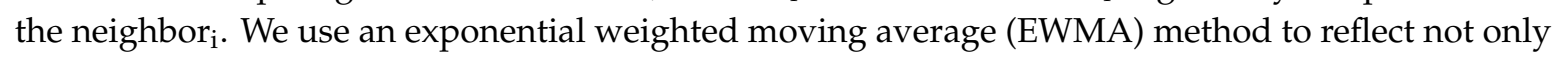
network connectivity but also cache effect in MPRcost [17].

The MPR selection algorithm is shown in the Algorithm 1: MPR selection. When a device needs to remove or add an entry in the 1-hop and 2-hop neighbor tables, then the MPR selection procedure is invoked. Foremost, MPR devices are elected among 1-hop neighbors of the device. Then, a device selects its MPR device that has the highest MPRcost between neighbors. After selection of the MPR, all 2-hop neighbors that are connected through MPR are removed from the 2-hop neighbor list. When a device finds a remaining 2-hop neighbor, then additional MPR devices are elected until the 2-hop neighbor list is empty. If the device does not have any remaining 2-hop neighbors, then the selected MPR list is notified through NBR-TL in the CA message. Thus, every device in the network can decide the device type, which can be a MPR or PMPR through the NBR-TL of the CA message from the neighbor(s). Figure 3 shows an example of the MPR selection using CA packets. In Figure $5 \mathrm{a}$, device 0 selects MPR devices 1 and 4 to cover its 2-hop neighbors of devices 2, 3, 7, and C. Following the same reason, every device selects its own MPR set as Figure $5 b, c$, respectively. After MPR selection, the entire MPR set of the topology is selected as Figure $5 \mathrm{~d}$. All broadcast messages are relayed only by the MPR devices. As a result, TOP-CCN can alleviate broadcast storming and duplicated transmission problems.

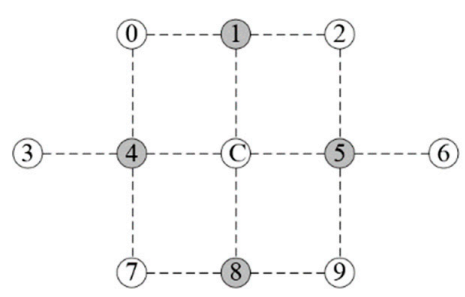

(a) MPR set selected by node $0,2,7,9, \mathrm{C}$

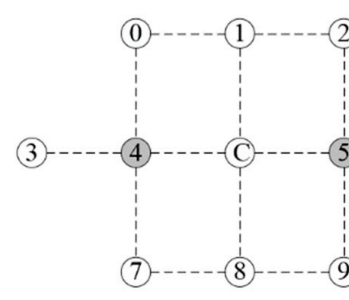

(c) MPR set selected by node 3,6

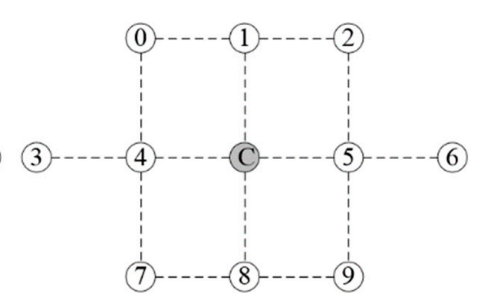

(b) MPR set selected by node $1,4,5,8$

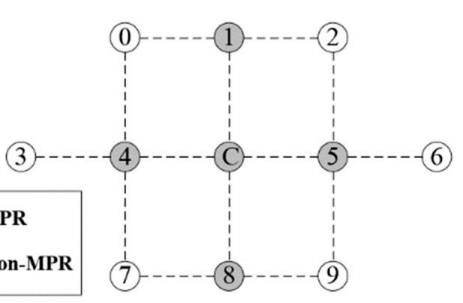

(d) Selected MPR set of the topology

Figure 5. An example of Multiple Point Relay (MPR) selection for reducing packet flooding of the CCN. 


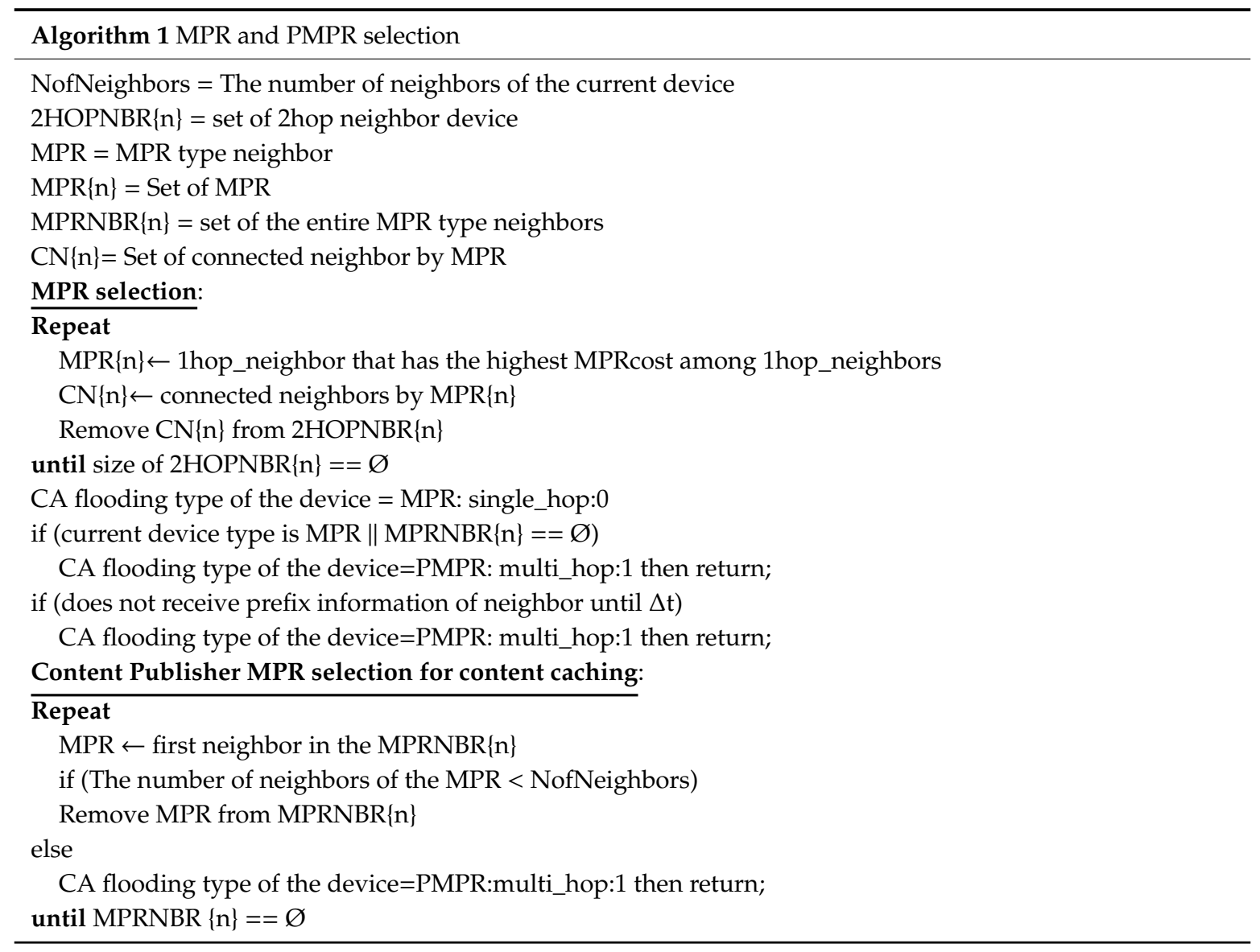

\subsection{PMPR-Based Aggregated Content (Prefix) Announcement and Content Caching}

In TOP-CCN, a CA packet has two types of prefix information such as content prefix of the sender and the neighbor content prefix list of the sender. When a device receives a CA packet, the content prefix list and the neighbor prefix list in the CA are inserted to FIB with received face information. In addition, 1-hop and 2-hop neighbor tables are updated using NBR-L, NBR-TL, and NBR-PL. Thus, a neighbor who receives a CA packet from its 1-hop or multi-hop neighbors can get the several prefix information of the neighbors of the sender. However, the proactive CA flooding increases network overhead such as packet collision, and congestion. To reduce the number of CA packet flooding, TOP-CCN selects PMPR devices who are allowed to broadcast multi-hop CA messages and can cache the data that is received from the AMI gateway. If the flooding type attribute of the received CA has single-hop value of 0 , then CA is not rebroadcasted. Multi-hop CA is generated only by the PMPR who has the largest number of neighbors among 1-hop MPRs. If the MPR device does not receive its neighbors prefix information during $\Delta \mathrm{t}$, then the MPR is changed as PMPR to notify neighbor(s) prefix information to the network. Therefore, content prefix information of the entire content provider in the network can be announced by only few multi-hop CA messages generated by PMPR.

The PMPR selecting algorithm is started by changing the 1-hop and 2-hop table information, and then deals with three cases as shown in Algorithm 1: Content Publisher MPR selection for content caching. The first is the absolute case. If a MPR device cannot find any MPR neighbor in its 1-hop neighbor table, then the MPR device itself becomes a PMPR for broadcasting multi-hop CA packet to the network. The second is the recovery case. If the MPR device cannot find content prefixes of the neighbor(s) from received CA packets during $6 \mathrm{~s}$, then MPR also becomes a PMPR to notify neighbor(s) prefixes. The third is the competition case. When a MPR finds neighbor MPR list from its 1-hop neighbor table, it compares the number of neighbors between MPR and MPR list. If the MPR has the largest number of neighbors among all the neighbors, the MPR device will become 
the PMPR. Figure 6 shows an example of PMPR selection. In this figure, PMPR set is $4, C$, and 5 because they have the largest number of neighbors between each neighbor MPRs. Therefore, only three devices in the network generate multi-hop CA messages. In addition, a multi-hop CA message is only relayed through MPR devices in the network. As a result, network overhead of the proactive content announcement is reduced., because the number of devices that makes the content announcement is also effectively reduced.

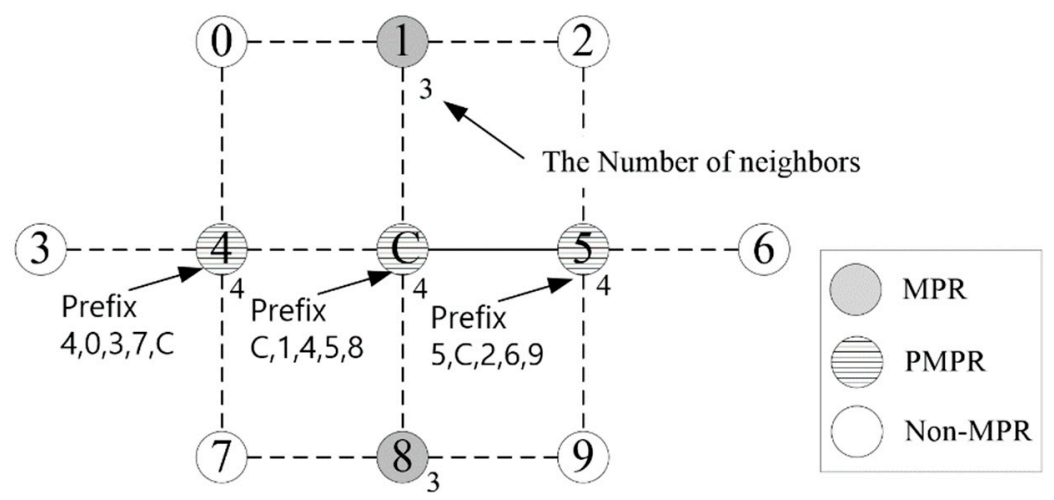

Figure 6. An example of Publisher MPR (PMPR) selection and aggregated prefix information of the multi-hop Content-prefix Announcement (CA) in TOP-CCN.

\subsection{Expected Hop Based Flooding Control of the CCN Packet}

Generally, CCN packets are flooded to the entire network using FIB and PIT information for reliable content requests and deliveries. However, the unlimited packet flooding induces high network congestion, which is critical in wireless SG environments. To solve this problem, hop distance between content provider and consumer is utilized. Expected hop distance between the packet sender and the destination such as content provider and consumer is measured by multi-hop CA messages from the PMPR. When a device receives a multi-hop CA message from its neighbor(s), the receiver device updates the content prefix, outgoing face, and the expected HOP count information in the FIB table. Thus, the expected hop attribute of the interest and content packets can be filled using expected hop information in the FIB. If the device receives an interest or content packet from its neighbor, then it compares the expected hop count value between FIB and received packet. If the device has a same or lower expected hop count, packet can be flooded into the network. When the expected hop count of the packet is lower than its own hop count, the packet is discarded to limit flooding.

\section{Performance Evaluation}

In this section, we present the performance of our proposed TOP-CCN using the ns-3 simulator [18]. Performance parameters such as reduced content dissemination delay, control message overhead, and increased accuracy of the face information in the PIT and FIB are measured and compared with flooding based CCN and RPL [19] in SG environment. Simulation parameters and smart grid application set used for the simulation are summarized in Table 1. In this table, density level 0.1 represents the one smart meter is placed in $100 \mathrm{~m}^{2}$ area and 100 smart meters are deployed in 1000 by $1000 \mathrm{~m}^{2}$ area. When a density level is set to 0.9 , then 900 smart meters are installed in a same region. These density level factor can be utilized to control collision rate between devices in a network. For example, Aggregated LMN environment model as apartment environment (aggregated electricity metering box), density of smart meter can be increased to 0.9 level density. As the network density increases, the chance of packet collision and interference are also increased. The performance evaluation is divided into 3 sections. First, we evaluate flooding range control scheme with ns-3 visualization tool. Second evaluation is reliability comparison between IPv6 based routing, CCN and TOP-CCN. This evaluation can show that $\mathrm{CCN}$ can be applied to LMN and outperform IP based wireless system. In a last evaluation part, we 
evaluated CCN caching performance between traditional CCN schemes and TOP-CCN. In this section we focus on $\mathrm{CCN}$ performance as content availability, smart meter firmware downloading time, etc.

Table 1. Simulation parameters and Smart Grid (SG) application set.

\begin{tabular}{|c|c|c|c|c|}
\hline \multicolumn{2}{|c|}{ Parameter } & \multicolumn{3}{|c|}{ Value } \\
\hline \multicolumn{2}{|c|}{ MAC/PHY protocol } & \multicolumn{3}{|c|}{ IEEE 802.15.4 SUN non-beacon mode } \\
\hline \multicolumn{2}{|c|}{ PHY rate/max PPDU size } & \multicolumn{3}{|c|}{250 Kb/s (DSSS)/2047 octets (SUN) } \\
\hline \multicolumn{2}{|c|}{ \# of MAC retransmissions } & \multicolumn{3}{|c|}{3} \\
\hline \multicolumn{2}{|c|}{ \# of AMI devices } & \multicolumn{3}{|c|}{$100-900$} \\
\hline \multicolumn{2}{|c|}{ Device density (\# of Devices per $100 \mathrm{~m}^{2}$ ) } & \multicolumn{3}{|c|}{$0.1-0.9$} \\
\hline \multicolumn{2}{|c|}{ Transmission/Interference range } & \multicolumn{3}{|c|}{$100 \mathrm{~m} / 140 \mathrm{~m}$} \\
\hline \multicolumn{2}{|c|}{ Network size/topology } & \multicolumn{3}{|c|}{1000 by 1000 m/Grid topology } \\
\hline \multicolumn{2}{|c|}{ MPRcost parameters } & \multicolumn{3}{|c|}{$\Delta \mathrm{t}: 10 \mathrm{~s}, \alpha: 0.5$} \\
\hline \multicolumn{2}{|c|}{ CA interval } & \multicolumn{3}{|c|}{$2 \mathrm{~s}$} \\
\hline \multicolumn{2}{|c|}{ Simulation Time } & \multicolumn{3}{|c|}{$1 \mathrm{~h}$} \\
\hline \multicolumn{2}{|c|}{ RPL parameter (DIO interval) } & \multicolumn{3}{|c|}{$1 \mathrm{~s}$ (evaluation for CCN and IP LMN) } \\
\hline Application & Data Size (Bytes) & $\begin{array}{c}\text { Delay } \\
\text { Requirement }\end{array}$ & $\begin{array}{l}\text { Refresh } \\
\text { Interval }\end{array}$ & Direction \\
\hline Time synchronization & 64 & $2 \mathrm{~s}$ & A content/Day & DOWN \\
\hline Price alert & 60 & $5 \mathrm{~s}$ & $300 \mathrm{~s}$ & DOWN \\
\hline Firmware update & 50,000 & Best effort & Months & DOWN \\
\hline Load control & 60 & $5 \mathrm{~s}$ & $300 \mathrm{~s}$ & DOWN \\
\hline Smart meter (on-demand) & 64 & Best effort & A content $/ 1 \mathrm{~h}$ & UP \\
\hline Smart meter (Periodic) & 64 & Best effort & $60 \mathrm{~min}$. & UP \\
\hline
\end{tabular}

\subsection{Evaluation of the Flooding Range Control Scheme}

In multi-hop wireless LMN environment, control messages can be flooded to entire LMN. This flooding message will increase network congestion because collision detection channel access scheme cannot be effectively adapted to wireless environment. Thus, wireless CCN have to reduce the number of flooding messages and its flooding range. Figure 7 shows the flow snapshot of flooding packets in each scheme, which are pure CCN, TOP-CCN without flooding range control and TOP-CCN with flooding control. A red circle represents a CCN node and a green arrow shows a packet flow. In Figure 7a, packet flows of pure CCN should fill out entire network to find the content provider and to reply the requested content. However, TOP-CCN as Figure $7 \mathrm{~b}$ dramatically reduces the number of flow by exploiting MPR and PMPR strategy. In this figure, the almost edge nodes in the network do not rebroadcast interest and content packet. In addition, flooding range is effectively limited through the expected hop count measured by CA messages from the all nodes.

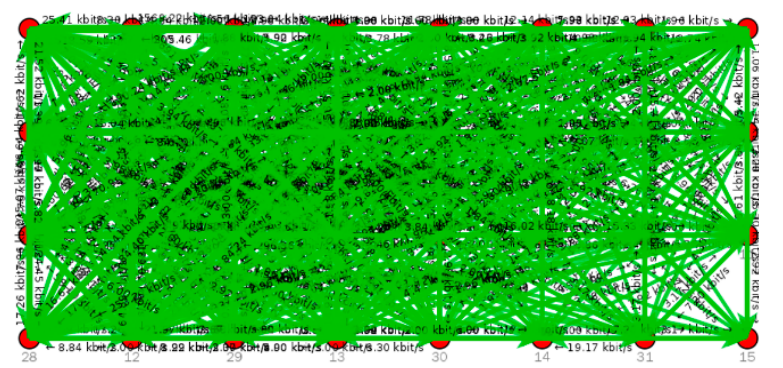

(a) Pure CCN over LMN environment

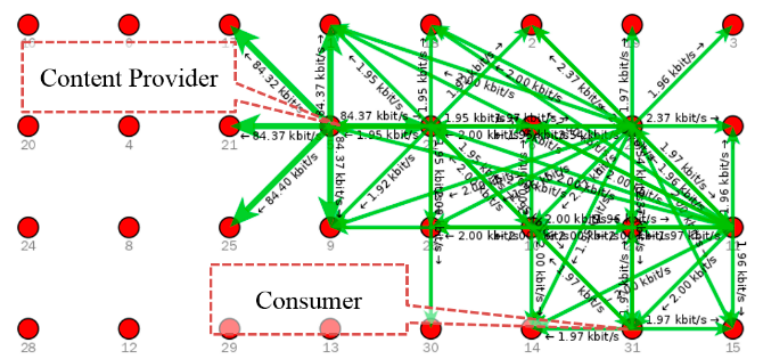

(b) Proposed scheme

Figure 7. The number of flooding messages (interest/content data) comparison in single consumer scenario using ns-3 visualization tool (Consumer: DCU/Gwateay, Content provider: Smart Meter device). 


\subsection{Evaluation of the Metering Application Reliability (Comparision between CCN and IP Protocols)}

In wireless multi-hop LMN environment, each meter can relay received metering packet. If the communication reliability is not stable between LMN group meters and DUC, multi-hop routing path can be utilized to improve its metering data reliability. Figure 8 shows the cumulative packet delivery ratio of the network. In this figure, the network density represents the number of devices in the specified network region. As seen in the figure, the performance of TOP-CCN with Flooding Control (FC) outperforms not only RPL but also CCN schemes, because the flooding control scheme reduce the CCN packet flooding in the wireless network. Furthermore, the selected PMPR AMI devices in the TOP-CCN can cache contents such as remote firmware update and peak price false alert from the SG server, which allows SG devices to receive these contents from intermediate devices instead of the server. This contributes to lesser hop distance that data has to traverse, which in turn reduces chance of collision and interference. The comparison of average hop distance between content location and device is shown in Figure 9. As aforementioned, the hop distance of the CCN schemes show outstanding performance compared to address based RPL protocol because SG devices can temporary cache various SG data and respond to content request from nearby devices in place of the server.

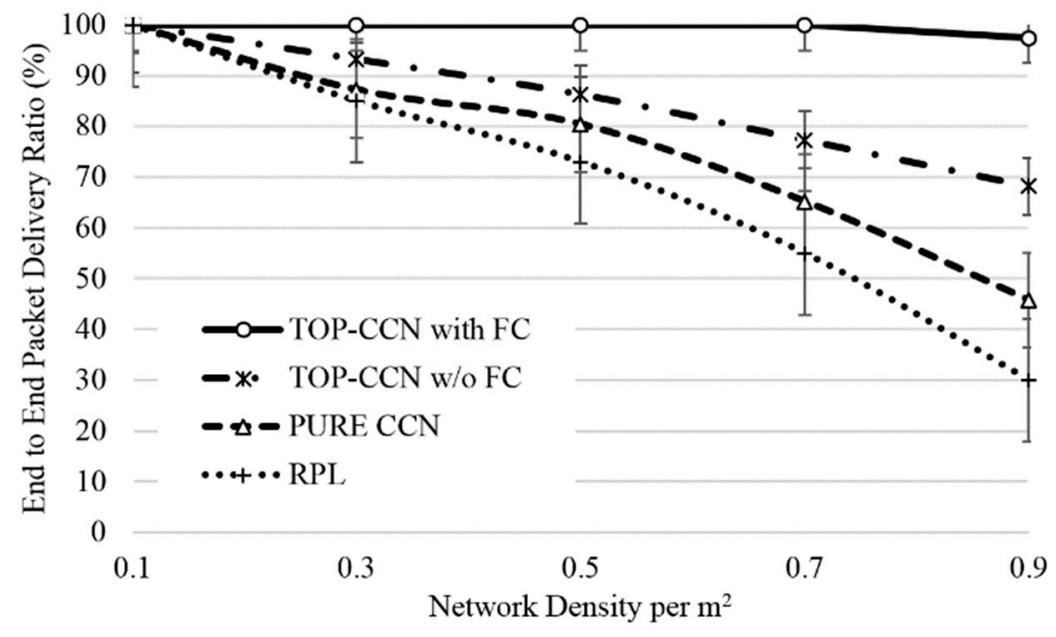

Figure 8. Cumulative end to end packet delivery ratio.

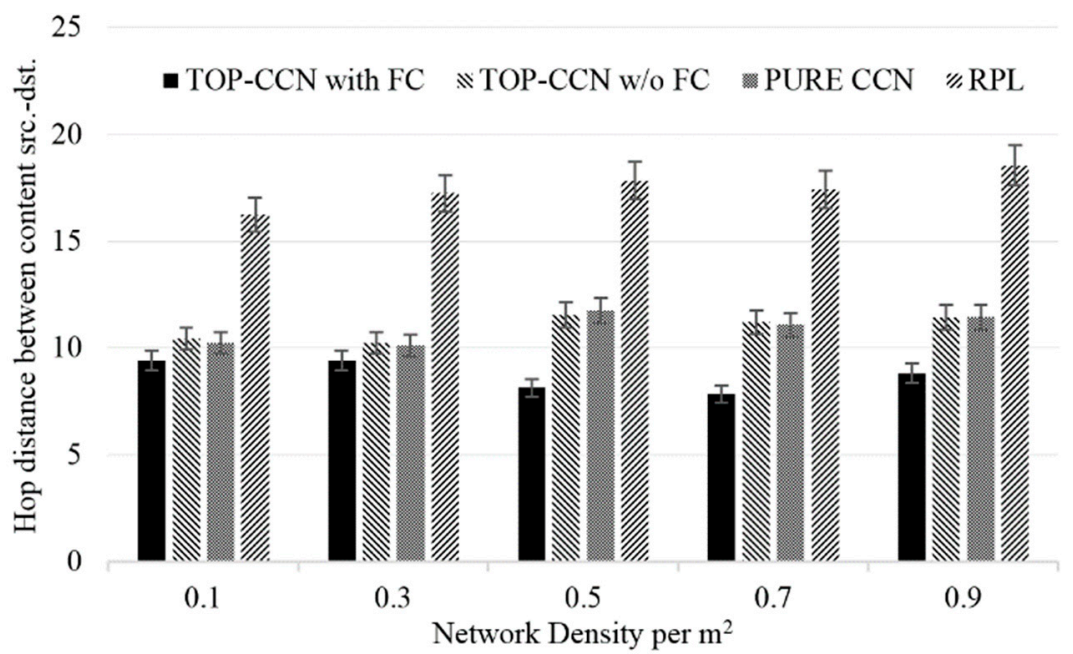

Figure 9. Average hop distance between content location and device.

When packets are lost due to collision, protocols such as IEEE 802.15.4 low rate MAC/PHY provides acknowledgement and retransmission algorithm to improve the transmission reliability between 1-hop neighbor devices. However, it is inefficient for the retransmission to frequently occur as 
it may also induce excessive overhead. Figure 10 shows the average MAC retransmission count of the device per packet transmission. In this result, the number of average retransmission counts is slightly increased depending on network density because the device is influenced by adjacent LMN devices. TOP-CCN with FC shows the least number of MAC retransmission because the number of hops and control packet transmission are reduced using MPR and PMPR devices. In overall, we can conclude that the proposed TOP-CCN can greatly increase reliability of SG network as well as reduce potential delay in end-to-end communication. This allows better servicing of various 1:M SG applications that can utilize CCN-based technique, as well as better services for existing IP-based monitoring data as the bandwidth usage and collision can be drastically reduced through TOP-CCN.

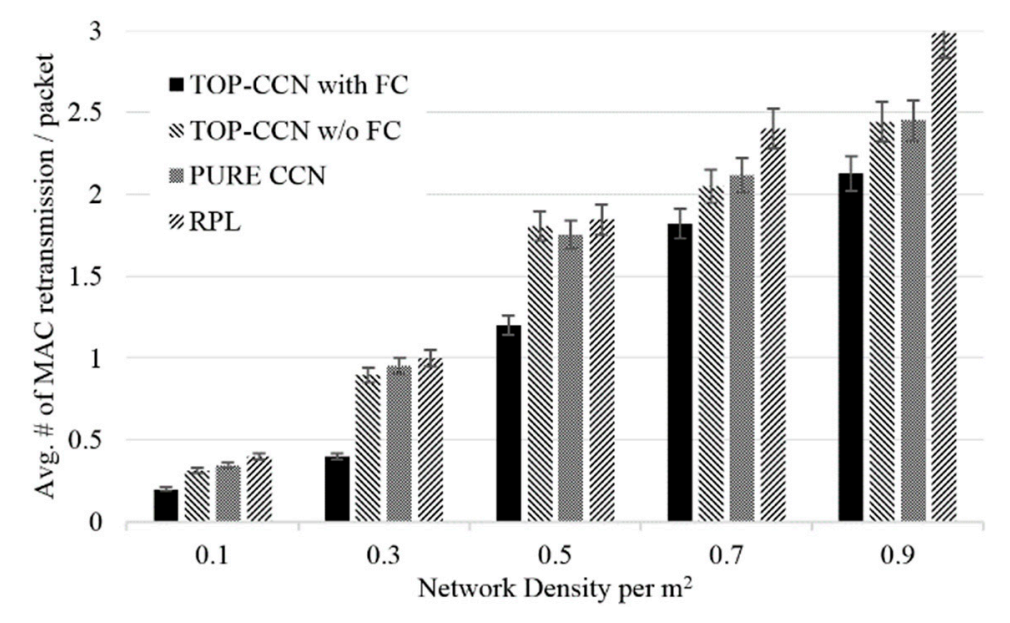

Figure 10. Average MAC retransmission per packet.

\subsection{Evaluation of the Metering Delay (Comparison between Traditional CCN Schemes)}

In smart metering infrastructure, end-to-end delay have to be optimized to metering depending on metering application requirement. Figure 11 shows the end-to-end delay of the various delay stringent SG applications such as time synchronization, price alert and load control traffic. In this result, the proposed TOP-CCN with FC shows better performance compared to other schemes, because data caching can considerably reduce average hop distance that a data must travel. We can observe that even though the network density increases, TOP-CCN with FC is the only technique that can guarantee less than $2 \mathrm{~s}$ end-to-end delay, which is the requirement time synchronization and time alert applications.

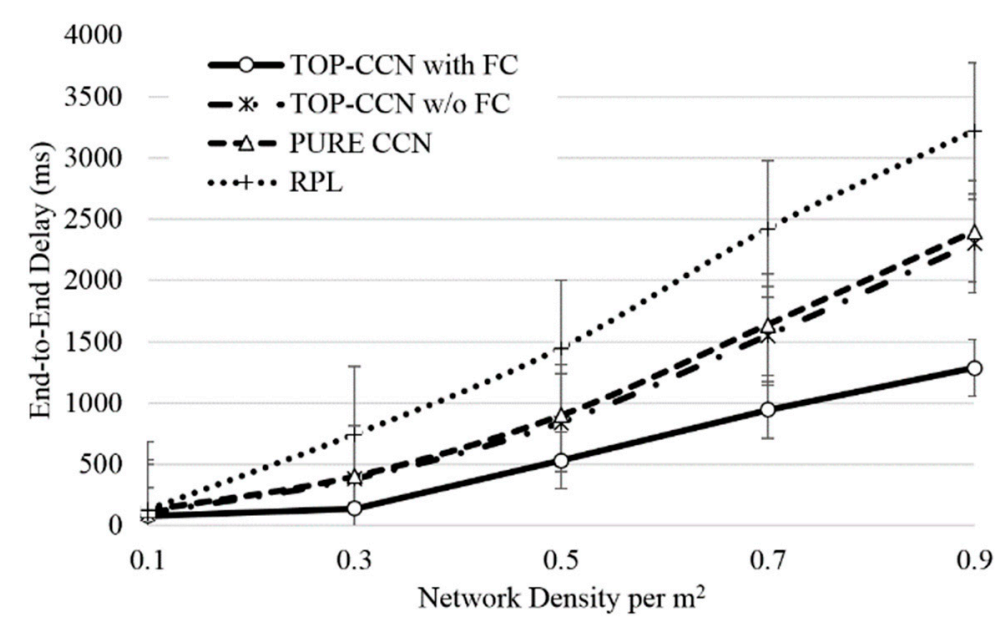

Figure 11. End-to-end delay of the delay limited applications. 
Figure 12 shows the content downloading time of remote firmware update file in the SG network. The size of firmware update content is $50 \mathrm{~Kb}$ and this file is fragmented by 10-byte unit chunks in the simulated CCN schemes. Thus all devices need to receive 5000 packets from the SG server. In the RPL scheme, all device in the network can receive the firmware content from the SG server by multicast and broadcast transmission. If some of the devices do not receive the transmitted firmware packet then retransmission procedure occurs in the application layer. This retransmission procedure increases the average hop distance, network congestion and interference of the network. However, the proposed TOP-CCN based architecture can eliminate application layer retransmission by utilizing the cache within SG devices. Thus, the content downloading time of the TOP-CCN is dramatically reduced compared to address based RPL protocol.

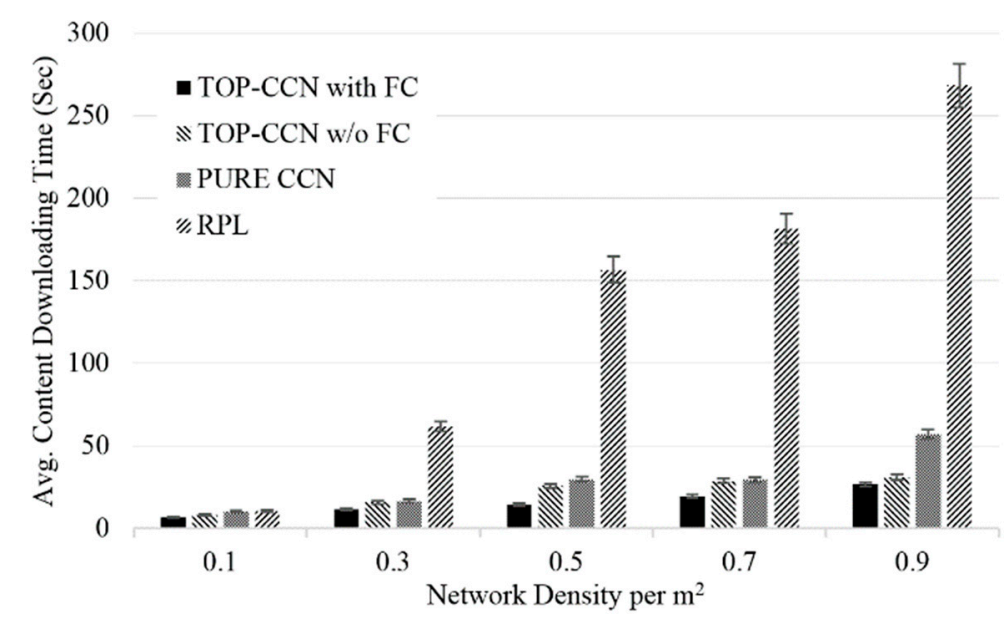

Figure 12. Average content downloading time of the BestEffort applications.

\subsection{Content Availability Comparision in Lossy Newtork Environment}

Content availability represents the accuracy of the face information, which is deeply depending on the network connectivity changing rate. When a node receives an interest packet from one of its neighbors, the incoming face information is added to reply the corresponding content packet to sender. However, in harsh network environments, incoming face information is not stable due to the link disconnections. Figure 13 shows the average content availability depending on the network instability. In this simulation we use the node mobility that can increase Face information instability. In this figure, TOP-CCN shows the highest content availability through CA based topology information update. If a node does not receive any CA packet from the connected neighbor during $6 \mathrm{~s}$, the corresponding face information of a neighbor is removed to prevent packet loss and transmission overhead. The performance of TOP-CCN with flooding range control algorithm is slightly decreased through the limited interest and content packet flooding. Figure 14 shows the network wide overhead of the request message. In this figure, $\mathrm{CCN}$ over MANET shows total $8 \mathrm{Mb} / \mathrm{s}$ network overhead due to the uncontrolled message flooding. E-CHANET shows the highest performance until five consumers due to the overhearing and interest aggregation algorithm. However, its performance decreases in high congestion environment due to inaccuracy of the content availability. Thus, interest retransmission is dramatically increased in E-CHANET. Even if TOP-CCN uses the proactive CA approach, it shows the lowest overhead as $2.4 \mathrm{Mb} / \mathrm{s}$ in 15 pair meter and DCU environments due to the increased content availability. The average content downloading time is shown in Figure 15. The performance is measured by a summation of 10 packet's delay because a metering content item is fragmented into 10 chunks. The delay performance of the E-CHANET has the smallest delay until 5 users smart meter firmware download (5 consumers) scenario. However, 10 and 16 firmware download environments, TOP-CCN with flooding range control shows the lowest delay due to the increased content availability and reduced network overhead. 


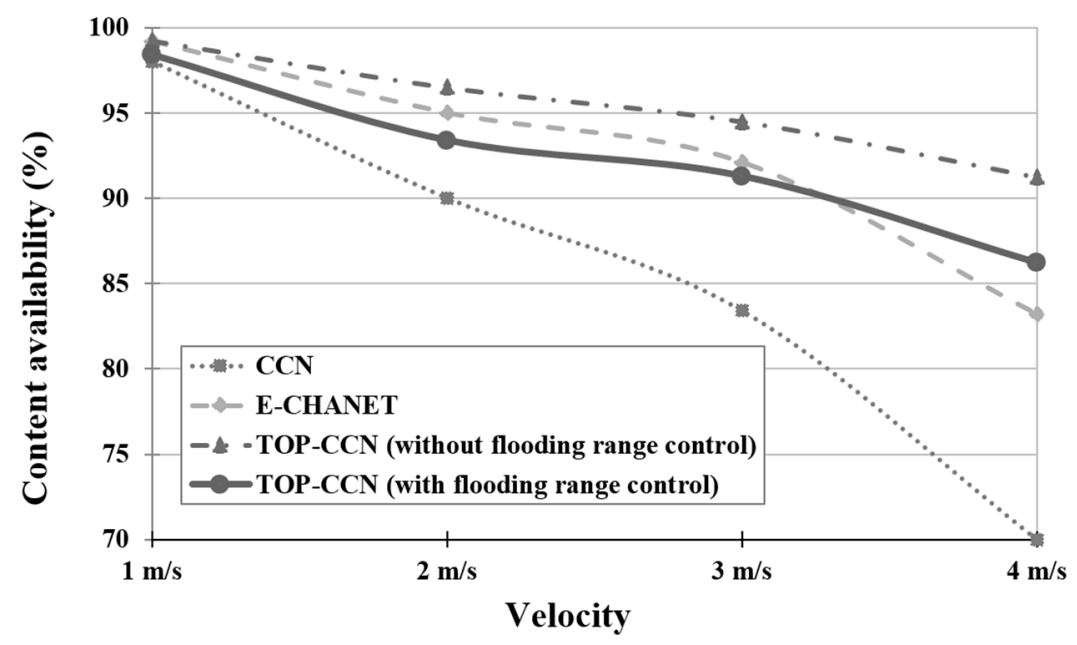

Figure 13. Content availability comparison depending on different connectivity instability levels.

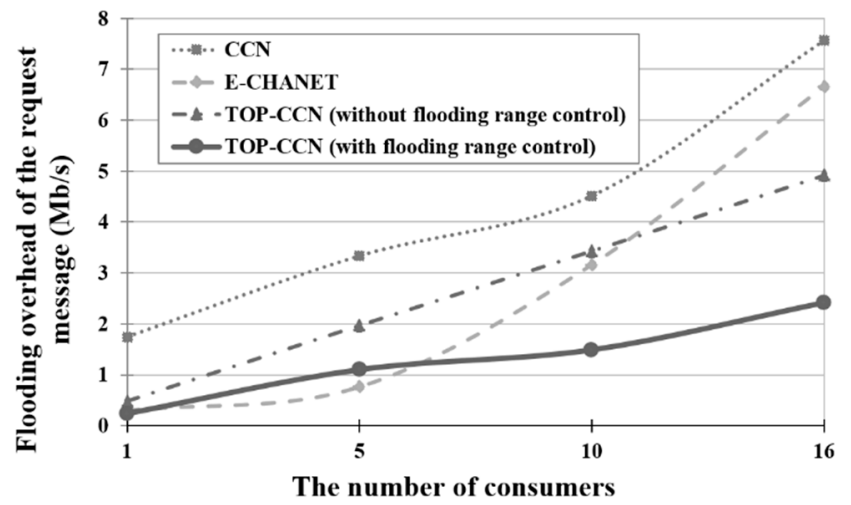

Figure 14. Flooding message overhead comparison depending on different number of firmware download users.

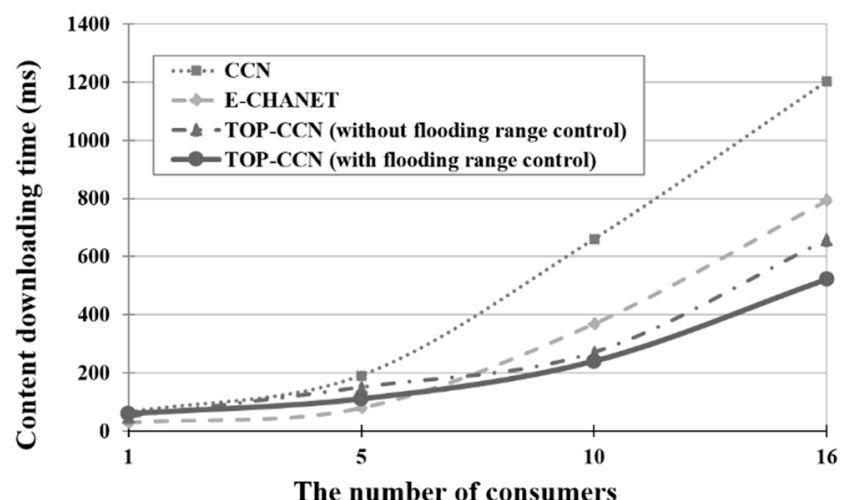

Figure 15. Meter firmware downloading time comparison depending on different number of firmware download users.

\section{Conclusions}

The future of $\mathrm{CCN}$ is very promising, as it is becoming the next-generation networking technology for efficient content dissemination in various application domains. Anticipating this trend, we present the method of applying CCN in wireless LMN through the topology aware CCN algorithm for Smart Grid. In this paper we presents naming and system model to transmit IP based Smart Grid to CCN based model, and evaluate directly between IP based and non-IP CCN based Smart Grid DLMS applications. This AMI CCN communication model can be applied to wired communication 
and wireless communication protocols. Also, we proposed flooding message mitigation algorithms for CCN that can reduce the channel access overhead of traditional CCN in wireless environment. The simulation result shows that our proposal guarantees improved packet delivery ratio, decreased content delivery time, hop distance, and MAC retransmission overhead compared to not only pure $\mathrm{CCN}$ but also RPL protocol that is widely considered to be enabled in smart utility networks. Based on our performance evaluation results, CCN can effectively support stable metering data collection in IEEE 802.15.4-2015 SUN wireless communication environment. We believe that our work can become a novel smart metering network model and guidelines for future studies regarding utilization and potential implementations of CCN in wireless smart grid LMN.

Author Contributions: Conceptualization, J.K.; methodology, J.K., B.-S.P., Y.-u.P.; software, J.K.; validation, J.K.; formal analysis, Y.-u.P.; investigation, B.-S.P.; resources, B.-S.P.; data curation, Y.-u.P.; writing-original draft preparation, J.K.; writing - review and editing, Y.-u.P.; visualization, J.K.; supervision, Y.-u.P.; project administration, Y.-u.P.; funding acquisition, J.K., Y.-u.P., and B.-S.P.

Funding: This research received no external funding.

Conflicts of Interest: The authors declare no conflict of interest.

\section{References}

1. Luan, W.; Sharp, D.; Lancashire, S. Smart grid communication network capacity planning for power utilities. In Proceedings of the IEEE PES T\&D 2010, New Orleans, LA, USA, 19-22 April 2010; pp. 1-4.

2. Kong, P.Y. Wireless neighborhood area networks with QoS support for demand response in smart grid. IEEE Trans. Smart Grid 2015, 7, 1913-1923. [CrossRef]

3. Yu, K.; Zhu, L.; Wen, Z.; Mohammad, A.; Zhou, Z.; Sato, T. CCN-AMI: Performance evaluation of content-centric networking approach for advanced metering infrastructure in smart grid. In Proceedings of the 2014 IEEE International Workshop on Applied Measurements for Power Systems Proceedings (AMPS), Aachen, Germany, 24-26 September 2014; pp. 1-6.

4. Jacobson, V.; Smetters, D.K.; Thornton, J.D.; Plass, M.F.; Briggs, N.H.; Braynard, R.L. Networking named content. In Proceedings of the 5th international conference on Emerging networking experiments and technologies (CoNEXT '09), Rome, Italy, 1-4 December 2009; pp. 1-12.

5. Kim, J.; Shin, D.; Ko, Y.B. TOP-CCN: Topology aware content centric networking for mobile ad hoc networks. In Proceedings of the 2013 19th IEEE International Conference on Networks (ICON), Singapore, 11-13 December 2013; pp. 1-6.

6. Mochizuki, K.; Obata, K.; Mizutani, K.; Harada, H. Development and field experiment of wide area Wi-SUN system based on IEEE 802.15.4g. In Proceedings of the 2016 IEEE 3rd World Forum on Internet of Things (WF-IoT), Reston, VA, USA, 12-14 December 2016; pp. 76-81.

7. Viswanath, S.K.; Yuen, C.; Tushar, W.; Li, W.T.; Wen, C.K.; Hu, K.; Chen, C.; Liu, X. System design of the internet of things for residential smart grid. IEEE Wirel. Commun. 2016, 23, 90-98. [CrossRef]

8. Mahadevan, P.; Uzun, E.; Sevilla, S.; Garcia-Luna-Aceves, J.J. CCN-KRS: A key resolution service for CCN. In Proceedings of the 1st ACM Conference on Information-Centric Networking (ACM-ICN '14), Paris, France, 24-26 September 2014; pp. 97-106.

9. Carofiglio, G.; Morabito, G.; Muscariello, L.; Solis, I.; Varvello, M. From content delivery today to information centric networking. Comput. Netw. 2013, 57, 3116-3127. [CrossRef]

10. Kumar, K.; Radhakrishnan, M.; Sivalingam, K.M.; Seetharam, D.P.; Karthick, M. Comparison of publish-subscribe network architectures for smart grid wide area monitoring. In Proceedings of the 2012 IEEE Third International Conference on Smart Grid Communications (SmartGridComm), Tainan, Taiwan, 5-8 November 2012; pp. 611-616.

11. Bastos, I.V.; Moraes, I.M. A forwarding strategy based on reinforcement learning for Content-Centric Networking. In Proceedings of the 2016 7th International Conference on the Network of the Future (NOF), Buzios, Brazil, 16-18 November 2016; pp. 1-5.

12. Oh, S.Y.; Lau, D.; Gerla, M. Content centric networking in tactical and emergency manets. In Proceedings of the 2010 IFIP Wireless Days, Venice, Italy, 20-22 October 2010; pp. 1-5. 
13. Amadeo, M.; Campolo, C.; Molinaro, A.; Ruggeri, G. Content-centric wireless networking: A survey. Comput. Netw. 2014, 72, 1-13. [CrossRef]

14. Sharma, P.; Souza, D.; Fiore, E.; Gottschalk, J.; Marquis, D. A case for manet-aware content centric networking of smartphones. In Proceedings of the 2012 IEEE International Symposium on a World of Wireless, Mobile and Multimedia Networks (WoWMoM), San Francisco, CA, USA, 25-28 June 2012; pp. 1-6.

15. Gungor, V.C.; Sahin, D.; Kocak, T.; Ergut, S.; Buccella, C.; Cecati, C.; Hancke, G.P. Smart grid technologies: communication technologies and standards. IEEE Trans. Ind. Inform. 2011, 7, 529-539. [CrossRef]

16. Tsado, Y.; Gamage, K.A.; Lund, D.; Adebisi, B. Performance analysis of variable Smart Grid traffic over ad hoc Wireless Mesh Networks. In Proceedings of the 2016 International Conference on Smart Systems and Technologies (SST), Osijek, Croatia, 12-14 October 2016; pp. 81-86.

17. Smith, T.H.; Boning, D.S. A self-tuning EWMA controller utilizing artificial neural network function approximation techniques. IEEE Trans. Compon. Packag. Manuf. Technol. Part C 1997, 20, 121-132. [CrossRef]

18. Mastorakis, S.; Afanasyev, A.; Zhang, L. On the evolution of ndnSIM: An open-source simulator for NDN experimentation. ACM SIGCOMM Comput. Commun. Rev. 2017, 47, 19-33. [CrossRef]

19. Ancillotti, E.; Bruno, R.; Conti, M. The role of the RPL routing protocol for smart grid communications. IEEE Commun. Mag. 2013, 51, 75-83. [CrossRef]

(C) 2019 by the authors. Licensee MDPI, Basel, Switzerland. This article is an open access article distributed under the terms and conditions of the Creative Commons Attribution (CC BY) license (http://creativecommons.org/licenses/by/4.0/). 